\title{
Research on Performance of Pineapple Leaf Fiber Healthy Underwear
}

\author{
Jinhe Gao ${ }^{1, a}$, Zhikai Zhuang ${ }^{1, b}$, Mingfu Li ${ }^{1, c}$, jin Zhang ${ }^{\text {1,d }}$, Wenwei Lian ${ }^{1, e}$ \\ ${ }^{1}$ Tropical Agricultural Machinery Research Institute, Chinese Academy of Tropical Agricultural \\ Sciences (CATAS), Zhanjiang 524091, Guangdong, P.R. China
}

ajinheg@sina.com, bzzk_119@sina.cn, ${ }^{\mathrm{b}}$ limingfu378@163.com, ${ }^{\mathrm{d}} \mathrm{zhjin1234@163.com}$

\begin{abstract}
Keywords: pineapple leaf fiber; refined dry jute; blended yarn; underwear; water absorption and quick dryness

Abstract. With pineapple leaf fiber as the raw material, refined dry jute is obtained through refined processing, which is blended with cotton to trail-produce $30 \mathrm{~S}$ pineapple leaf fiber/cotton (30/70) blended yarn. Through the combination of industry, university and research and by cooperating with the textile enterprises, pineapple leaf fiber healthy underwear is researched, developed and trial-produced, and its water absorption and quick dryness are examined. The results show that the water absorption and moisture permeability of pineapple leaf fiber underwear reach the indicators of national textile standard, which provides a theoretical basis for the research and development of other pineapple leaf fiber products in the following.
\end{abstract}

\section{Introduction}

After fruit picking of pineapple, about 5-10t pineapple leaves will be left per $\mathrm{Mu}$, the traditional processing method is burning or smashing for farmland fertilization, which causes great resource waste and environmental pollution. The fiber extracted from pineapple leaves at present is called pineapple leaf fiber, belonging to leaf fiber. It does not only have the general characteristics of cotton and jute, but also has excellent natural special functions like sterilization, odor removing and mite driving, and it is also superior to other jute fibers in terms of coolness, moisture absorption and release, air permeability, softness, silky gloss and non-itching feeling. The main components of textile products in the market like underwear are cotton, viscose, chemical fiber and other fiber fabrics, and the products designed always emphasize the innovation in fabric, style, color and decoration, while ignoring the most important link, the healthy safety. Researches have shown that groin is a large sweat gland part of human beings; in summer, the sweat glad is of exuberant secretion and the protein contained in the sweat generates odor with the bacterial decomposition, and wearing underwear with poor moisture absorption and release and no air permeability is easier to cause skin diseases.

In this article, pineapple leaf fiber is blended with cotton to research, develop and trial-produce pineapple leaf fiber healthy underwear, which effectively solves the potential safety hazard described above, and the water absorption and moisture permeability of pineapple leaf fiber healthy underwear reach the indicators of national textile standards, providing a theoretical basis for the research and development of other pineapple leaf fiber products in the following.

\section{Sample trial production}

Preprocessing and blending of raw materials. The pre-opening pineapple leaf jute fiber has a great difference in length with certain doubling fiber and ultra-long fiber, in order to adapt to the production in cotton textile equipment, pre-opening is required to improve the fiber separation, so as to reduce the consumption of pineapple leaf fiber in the process of yarning, and meanwhile, it is required to appropriately adjust the technological parameters related to the opening machinery, so as to break some ultra-long fibers. The pre-opening is conducted on the waste cotton processing machine.

Oil feeding is to reduce the fiber stiffness, reduce winding caused by static electricity, enhance cohesive force and improve spinning property. The pre-opened pineapple leaf fiber is added with appropriate oil agent and water, stowed and maintained for $72 \mathrm{~h}$ before unpacking for use. 
The oil agent consists of cohesive agent, anti-static agent and softening agent. The cotton sorting proportion should meet the requirements of blending ratio. In consideration of similarity of pineapple fiber itself with other jutes in actual production and higher fiber purchase cost and falling consumption than cotton, during cotton sorting, the cotton sorting proportion should be increased appropriately. Upon test, it is appropriate that the feed ratio of pineapple leaf fiber/cotton is $30 / 70$ during bale blending.

\section{Knitting technology}

Technological process: yearn preprocessing-weaving-preprocessing-dyeing-washing and softening-drying and forming

Yarn preprocessing: the pineapple leaf fiber/cotton blended yarn has more hairs, with stiffness larger than cotton yarn, the yarn is applied with wax and meanwhile it is required to control the lodging direction of hair and ensure the opposite direction with yarn feeding during weaving, so as to be convenient for the smooth weaving.

Weaving processing: compared with the cotton yarn, the pineapple leaf fiber/cotton blended yarn has a small tension of yarn feeding during weaving, the ambient humidity is highly controlled, relative humidity is appropriate to be about $70 \%$, and the opening height is large.

\section{Dyeing and finishing process and sorting technology}

Pre-dyeing processing: the pineapple leaf fiber adopts half-degumming method, in addition to the defective gum, the contents of other impurities are also relatively high, therefore, on the basis of the traditional technological dyeing, the dyeing technology is improved.

Post-sorting technology: the post-sorting of pineapple leaf fiber knitted has the same purpose with the cotton knitted fabric, both are for the purpose of improving the hand feeling of fabric, enhancing the cloth cleanness and levelness, reducing shrinkage rate and improving the serviceability of fabrics. For this reason, the drying and pre-shrinking setting are tested and researched.

Loose tension-free drying machine is used for drying, during the drying process, the gray fabric is in loose state, and is fully shrunk, which is beneficial to reduce the shrinkage rate. Technological condition: temperature $90^{\circ} \mathrm{C}$; spinning wheel speed $10-14 \mathrm{~m} / \mathrm{min}$.

The pre-shrinking setting is of importance to improve the shrinkage rate, hand feeling and elasticity of the gray fabric; blanket pre-shrinking setting machine is used for three-excessive pre-shrinking setting, i.e. excessive feeding expansion, excessive feeding drying and excessive feeding calendaring. On the basis of repeated experiment, it is determined that it is appropriate when the calendaring width is $6 \mathrm{~cm}$ larger than the opening diameter of gray fabric. Technological condition: temperature $95-108^{\circ} \mathrm{C}$; spinning wheel speed $20-22 \mathrm{~m} / \mathrm{min}$, excessive feeding $10-15 \%$ and appropriate steam.

Evaluation of mechanical property of single fiber and water absorption and quick dryness of knitted fabric

\section{Mechanical property}

Tensile property. YG(B)008E-type single-fiber strength tester is used to test the tensile performance of fiber, with load test range $0-50 \mathrm{cN}$, clamping distance $10 \mathrm{~mm}$, tensile speed $10 \mathrm{~mm} / \mathrm{min}, \mathrm{RH}=60 \%$ and $5=25^{\circ} \mathrm{C}$.

Softness. The fiber softness is tested with twisting method, 1 bundle fiber is clamped to Y331 twisting meter, with a clamping distance of $300 \mathrm{~mm}$, and the softness is expressed with the number of twisting from twisting to breaking.

\section{Textile product performance test}

The performance indicators like water absorption, moisture permeability and air permeability are tested according to GB/T21655.1-2008 Textile-Evaluation of Water Absorption and Quick Dryness-Part 1: Single combination test method.

\section{Result and analysis}

Mechanical property. It can be seen from Table 1 that the pineapple leaf fiber has a high strength, small elongation, and large elastic modulus, and this is somewhat associated with the chemical 
components and micro-structure in the pineapple leaf fiber. The elongation of pineapple leaf fiber is between that of flax and jute. Generally, its fiber spinnability and yarning quality is also between the two, i.e. superior to jute while inferior to flax.

Table 1 Mechanical property of pineapple leaf fiber, flax and jute

\begin{tabular}{|c|c|c|c|c|}
\hline Fiber & Strength/cN.tex-1 & Elongation (\%) & $\begin{array}{l}\text { Elastic modulus } \\
\qquad / \times 107 \text { pa }\end{array}$ & $\begin{array}{c}\text { Softness /twist } \\
(20 \mathrm{~cm})\end{array}$ \\
\hline Pineapple leaf fiber & 31.26 & 4.21 & 10.26 & 192 \\
\hline Flax fiber & 46.57 & 4.86 & 9.36 & l \\
\hline Jute fiber & 25.36 & 3.96 & 11.25 & 93 \\
\hline
\end{tabular}

Textile performance. It can be seen from Table 2 that the water absorption, moisture permeability and air permeability of pineapple leaf fiber healthy underwear reach the indicators of national textile indicator, while the test measured from water dropping diffusion time is about 10 times of the standard value. The longer water dropping time might because the pineapple leaf fiber has longitudinal crack and pores on the surface, with a large specific surface area, loose and porous internally. Therefore, after water dropping, the diffusion speed is relatively slow. 
Table 2 Evaluation of water absorption and quick dryness of pineapple leaf fiber underwear

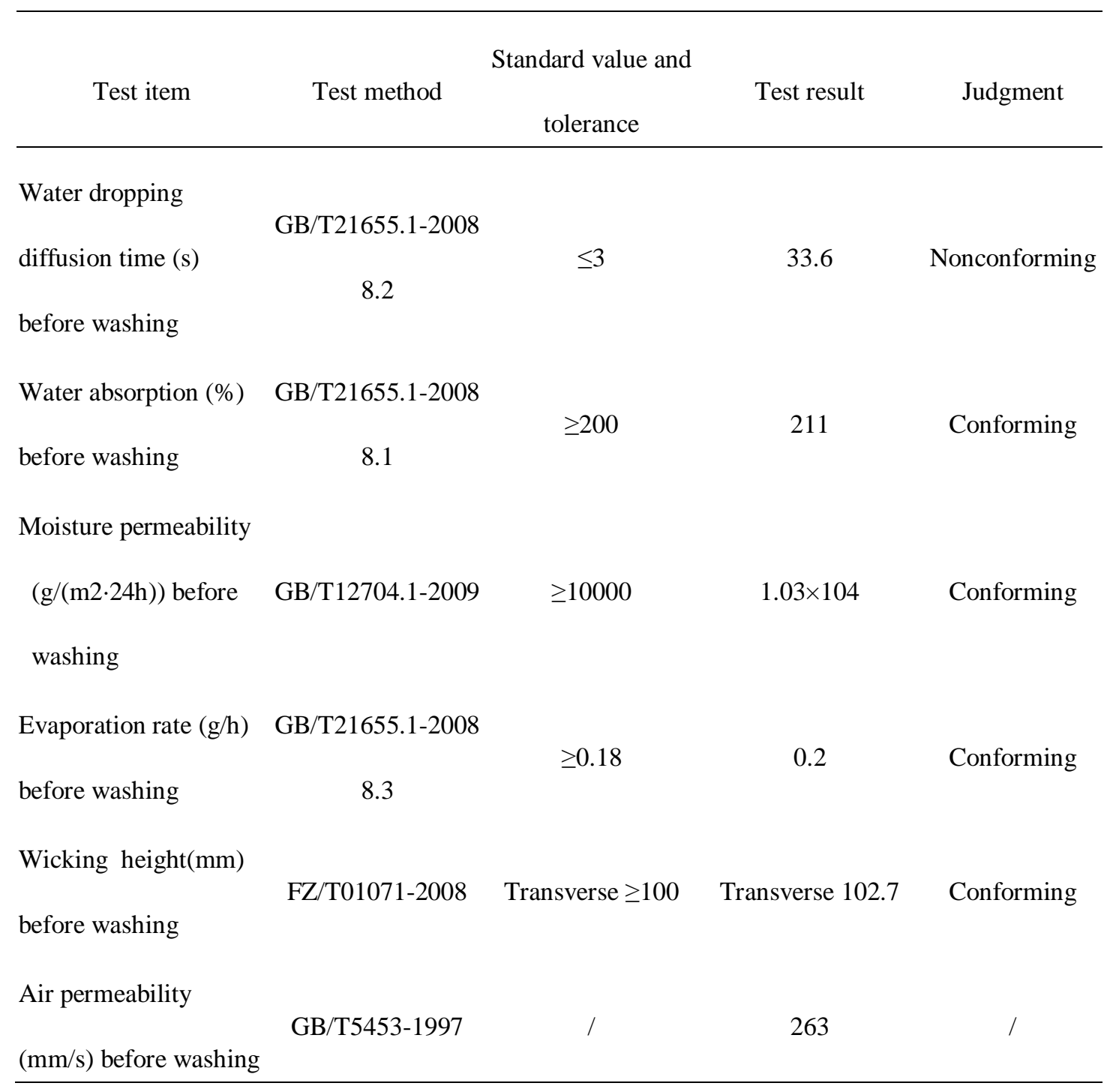

\section{Conclusion}

(1) The pineapple leaf fiber has its mechanical property approach to that of flax and jute. According to the test result, its spinnability and yarning quality should be inferior to flax while superior to jute. (2) The pineapple leaf fiber is in the form of half-degumming, and by improving the original knitting, dyeing and finishing and post-sorting technology, pineapple leaf fiber series products are trial produced.

(3) The water absorption and quick dryness of pineapple leaf fiber healthy underwear completely conform to the national textile standard, it does not only have a good moisture permeability, the fiber itself also has natural disinfection function, so it will be appreciated by the consumers.

\section{Acknowledgements}

This work was financially supported by the Fundamental Scientific Research Funds for Chinese Academy of Tropical Agricultural Sciences ( Project No.1630062015022). Major Science and Technology Projects of Hainan Province (ZDZX2013023-3). and the Special Fund for Agro-scientific Research in the Public Interest (NO. 201203021). 


\section{References}

[1] J.Zhang. Pineapple Leaves'Comprehensive Development \& Utilization [M]. Haikou: Nanhai Publishing Company, 2006.

[2] J.Zhang, M.F.Li, Z.Q.Ou, et al. . China Conference. 2003-10-01.

[3] W.W.Lian, J.Zhang, M.F.Li, et al. Initial Study on Pineapple Leaf Dregs As Silage to Raise Pigs. Guangxi Tropical Agriculture, 2002, 4

[4] M.F.Li, et al. Trends of Study on Pineapple Leaf Fiber and Development Countermeasures. Linen Weaving Technology.1999, 22 (1)

[5] J.Zhang, M.F.Li, W.W.Lian, et al. Journal of Chinese Agricultural Engineering. 2000, 16 (6): 99-103.

[6] T.Huang, J.M.Jiang, J.L.Wang, et al. Shanghai Textile Science \& Technology. 2009, 37: 9-12.

[7] B.Q.Shang, Q.H.Yuan, Y.M.Chen. Dyeing \& Finishing Technology. 2003, 25(6): 34-36.

[8] H.X.Zhou, S.H.Shu. Shanghai Silk, 1999, 1:7-9.

[9] X.F.Ye.Foreign Textile Technology: Chemical Fibers, Dyeing \& Finishing, Environmental Protection. 1996, 1: 1-4.

[10] R.Wang, D.S.Zhang. Synthetic Fiber Industry. 2002, 25(5): 44-46.

[11] X.S.Gao, Y.Yan, Y.Zhuang, J.Q.Hong. Synthetic Fiber Industry. 2000, 23(4): 35-38.

[12] M.Yao. Beijing: Textile Industry Press, 1997, 10.

[13] L.X.Gu,Z.F. Liu, et al. China Petrochemical Press, 1997, 2.

[14] LIY, HOLCOMBEBV.Textile Res, J, 1992, 62(4): 211-217.

[15] LIY, Z.X.Luo.Textile Inst, 2000, 21(2): 302-316. 